\title{
Experimental Methods Necessary in Acoustic Spectroscopy
}

\author{
B.B.J. LindE ${ }^{a, *}$ AND E.B. SkRODZKA ${ }^{b}$ \\ ${ }^{a}$ Institute of Experimental Physics, University of Gdańsk, Gdańsk, Poland \\ ${ }^{b}$ Institute of Acoustics, Adam Mickiewicz University, Poznań, Poland
}

\begin{abstract}
The paper is an overview of a theory and experimental methods used in acoustic spectroscopy. Theoretical discussion about the ultrasonic relaxation process related to energy transfer between translational and vibrational degrees of freedom as well as to the existing of chemical isomerism is presented. Two basic ultrasonic measurements methods of absorption and velocity in very wide frequency range from hundreds $\mathrm{kHz}$ to tens $\mathrm{GHz}$ are described. Several experimental examples of advantage of the ultrasonic spectroscopy are also shown.
\end{abstract}

DOI: 10.12693 /APhysPolA.127.120

PACS: 43.35.Bf, 43.35.Fj

\section{Introduction}

The acoustical spectroscopy is closely connected with molecular acoustics which is the study of molecules and their interactions using elastic waves of audio frequencies $(f<20 \mathrm{kHz})$, ultrasonic frequencies $(20 \mathrm{kHz}-1 \mathrm{GHz})$ and hypersonic frequencies $(f>1 \mathrm{GHz})$. When the elastic wave passes through a medium in which there exists the equilibrium, the effect on the equilibrium depends on the frequency of the wave. If the period of the elastic wave is much longer than the relaxation time for the alteration of the position of equilibrium, the later is disturbed by the wave. If the period of the wave is much shorter than the relaxation time, the wave does not "see" the equilibrium which consequently remains undisturbed. When the period is comparable to the relaxation time, changes in the propagation velocity and absorption coefficient of the elastic wave occur. From the measurements of the changes, the relaxation time and the rate constants of the equilibrium may be determined. Since the period of elastic wave can range from 1 to $10^{-10} \mathrm{~s}$, a wide range of equilibria can be studied.

\subsection{Sound waves in fluids, classical treatment}

From a macroscopic point of view, the following three factors may be expected to produce a time lag between pressure and density in a sound wave: viscosity [1], heat conduction [2], heat radiation [3].

The experimental evidence indicates that in most fluids the absorption is caused primarily by viscosity and heat conduction and that the effect of radiation is negligible $[4,5]$. In liquids, viscosity plays the major role. In gases viscosity and heat conduction come into play about equally, but they are often overshadowed by molecular phenomena.

Deriving the formula (1) for $\alpha_{1}$, it was assumed by Stokes that viscous losses were absent in motion of uniform compression so that the volume viscosity $\eta_{v}$ was taken to be zero. It is now known that this is not generally true, and inclusion of the volume viscosity in the

* corresponding author; e-mail: fizbl@univ.gda.pl hydrodynamic equations leads to a total viscous contribution

$$
\alpha^{\prime}=\frac{2 \pi^{2}}{\rho_{0} c_{0}^{3}}\left(\eta_{v}+\frac{4}{3} \eta_{s}\right) f^{2} .
$$

The difference between the measured absorption coefficient $\alpha$ and the classical one as well as the frequency dependence was given the rise to creation the new branch of physics - acoustic spectroscopy (investigation of acoustic relaxation processes).

\subsection{Relaxation}

In classical hydrodynamics the normal state equation for the particular fluid is used assuming that the state of the fluid is entirely determined by the knowledge of any two variables. We have already indicated that this hypothesis cannot be valid when particles may exist in various energetic states; the particles of the fluid (molecules) may either have internal degree of freedom differently excited or find themselves in different energetic conditions in relation to the neighbouring particles. In such a case, for each set of independent thermodynamic variables one has to consider a distribution of particles among the allowed energy states. When the state variables are changed the particles have to pass from one equilibrium distribution to another and this requires certain time. A characteristic time $(\tau)$ is introduced as the time required to the departure from a state of equilibrium to 1 /e of its original value.

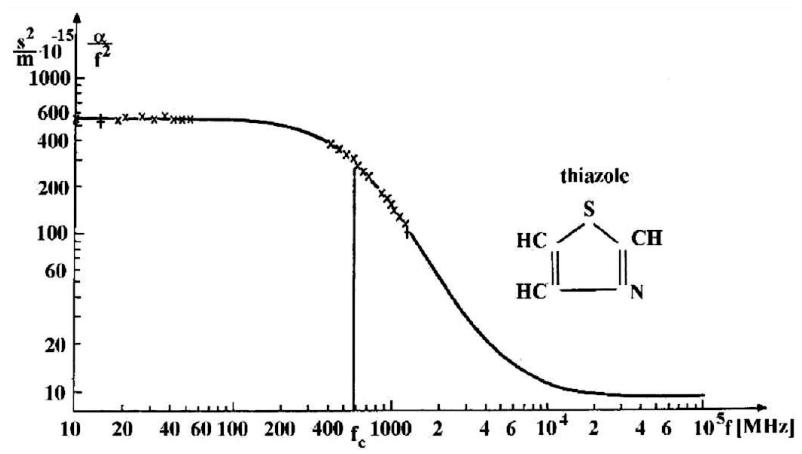

Fig. 1. Relaxation curves for thiazole, $\tau=2.8 \times$ $10^{-10}$ s [6]. 
In the case of acoustic relaxation $\alpha / f^{2}(f)$ absorption curve (Fig. 1) can be described as follows:

$$
\frac{\alpha}{f^{2}}=\frac{A}{1+\omega^{2} \tau^{\prime 2}}+B
$$

where $A, B-$ constants, $\tau^{\prime}=\tau\left(C_{p}-C_{i}\right) / C_{p}, C_{p}$ specific heat at constant pressure, $C_{i}$ - specific heat of vibrational degrees of freedom (Eq. (5)).

\subsection{Vibrational relaxation}

For the Kneser relaxation process, e.g. exchange of energy between translational and vibrational degrees of freedom, Eq. (2) has the form

$$
\frac{\alpha}{f^{2}} \approx \frac{2 \pi^{2}}{c_{0}} \frac{R^{\prime} C_{i}}{C_{v}\left(C_{p}-C_{i}\right)} \frac{\tau^{\prime}}{1+\omega^{2} \tau^{\prime 2}},
$$

where $C_{v}$ - specific heat at constant pressure, $c_{0}-$ ultrasonic velocity for low frequency.

In the low frequency range it can be rearranged giving

$$
\frac{\alpha}{f^{2}} \approx \frac{2 \pi^{2}}{c_{0}} \frac{R^{\prime} C_{i}}{C_{v}\left(C_{p}-C_{i}\right)} \frac{\tau^{\prime}}{1+\omega^{2} \tau^{\prime 2}}
$$

and

$$
C_{i \text { acoust }} \approx \frac{A c_{0}}{2 \pi^{2}} \frac{C_{p} C_{v}}{C_{p}-C_{v}} \tau_{\text {acoust }} .
$$

The contribution of molecules vibration to the specific heat may be also calculated from the vibrational energies distribution (frequencies $\nu_{i}$ ) using the Planck-Einstein formula [6]:

$$
C_{i \text { opt }}=R \sum_{i} g_{i}\left(\frac{h \nu_{i}}{k T}\right) \frac{\exp \left(-h \nu_{i} / k T\right)}{\left[1-\exp \left(-h \nu_{i} / k T\right)\right]^{2}},
$$

where $g$ - degree of degeneration, $\nu_{i}$ - frequency of the $i$ vibration mode, $T$ - temperature, $h$ - the Planck constant, $k$ - the Boltzmann constant.

Comparison of the values $C_{i \text { opt }}$ and $C_{i \text { acoust gives a }}$ possibility to decide which vibrational degrees of freedom take part at the observed relaxation process [7]. Usually all vibrational degrees of freedom take part in such a process [7-11]. The pressure spectroscopy proves this conclusion $[12,13]$.

\subsection{Isomeric relaxation}

In the case when molecules can occupy two or more internal energy levels the equilibrium distribution of molecules among these levels is perturbed by the passage of a sound wave. At sufficiently low sound frequencies, the relatively long interval between successive cycles allows plenty of time for energy to be transferred from the ultrasound wave to the internal degrees of freedom. As the ultrasound frequency increases, a time-lag occurs between the temperature and pressure variation in the wave and the adjustment of the internal equilibrium. It leads to a maximum in the ultrasonic absorption per wavelength and a decrease in $\alpha / f^{2}$. Figure 2 shows the energy level diagram for a two-state equilibrium (trans-isomer and cis-isomer)

$$
\boldsymbol{A}_{\text {trans }} \Leftarrow \begin{gathered}
k_{f} \\
k_{b}
\end{gathered} \Rightarrow \boldsymbol{B}_{\text {cis }}
$$

in which $\boldsymbol{B}$ is the higher energy state. Studies of liq- uids with ultrasonic longitudinal waves provide information on the rate constants for the forward and backward reactions, $k_{f}$ and $k_{b}$, the free energy barrier $\Delta G_{b}^{\prime}$, and the free energy difference between the two levels, $\Delta G$. These quantities are calculated from the variation of the absorption per wavelength $\mu$ with frequency and temperature. At a given temperature $\mu$ has a maximum value at a characteristic frequency $f_{c}$. From the temperature dependence of $f_{c}, \Delta G_{b}^{\prime}$ may be found, while the temperature dependence of $\mu_{\max }$ yields $\Delta G$. The theoretical basis of this calculation is outlined in the following paragraphs.

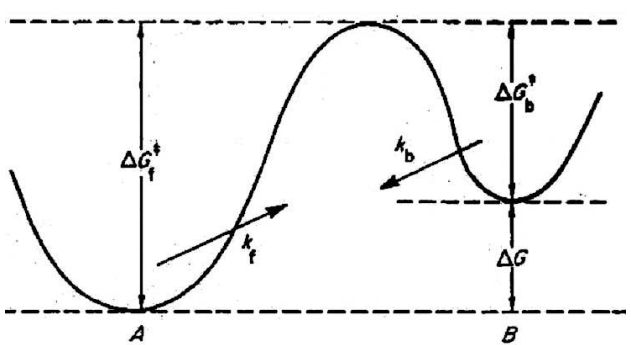

Fig. 2. Energy level diagram for two-state equilibrium.

\subsection{Determination of energy barriers}

Whenever we have the two-state equilibrium (Fig. 2), it is possible to assume that each reaction is unimolecular and take place in an ideal solution. The characteristic frequency $f_{c}$ of the equilibrium is related to the rate constants by the expression

$$
f_{c}=\frac{1}{2 \pi \tau}=\frac{k_{f}+k_{b}}{2 \pi},
$$

where $\tau-$ relaxation time of the equilibrium.

The theory of rate processes [14] expresses the rate constant $k_{r}$ of a reaction in terms of the increase in free energy $\Delta G^{\prime}$ on moving from the initial state to an intermediate activated state

$$
k_{r}=\frac{k T}{h} \exp \left(-\Delta G^{\prime} / R T\right) \text {. }
$$

When $\Delta G_{b}^{\prime} \ll \Delta G_{f}^{\prime}, k_{b} \gg\left\{k_{f}, k_{b}\right\}$ it is possible to obtain the

$$
k_{b}=2 \pi f_{m} \text {. }
$$

\subsection{Determination of the energy difference between two states}

From Eq. (2) and knowing that $\lambda=c / f$ we can write

$$
\mu=\frac{A c f}{1+\omega^{2} \tau^{\prime 2}} \text {. }
$$

For the rotational isomeric relaxation we are justified in neglecting the velocity dispersion $[15,5,16]$, which is less than $1 \%$. The absorption per wavelength achieves a maximum when $f=f_{c}$ :

$$
\mu_{\max }=\frac{1}{2} A c f_{c} .
$$

According to the theory of thermal relaxation we have 


$$
\mu_{\max }=\frac{\pi}{2} \frac{\kappa-1}{C_{p}} C_{i}^{\mathrm{r}} .
$$

$C_{i}^{\mathrm{r}}$ determines the heat absorbed by the reaction (5) when the temperature is increased. From the definition of internal molar heat

$$
C_{i}^{\mathrm{r}}=-\frac{\Delta H}{N_{A}}\left(\frac{\partial n_{A}}{\partial T}\right)
$$

we can derive, in the case of equilibrium

$$
C_{i}^{\mathrm{r}}=\frac{\Delta H^{2}}{R T^{2}} \frac{\exp (-\Delta G / R T)}{(1+\exp (-\Delta G / R T))^{2}} .
$$

We can assume that $\Delta G / R T>3$. Then the term between brackets may be approximated by one, with accuracy $\pm 5 \%$. From Eqs. (12) and (14) we obtain even better approximation

$$
\frac{2 \mu_{\max }}{\pi}=\frac{\kappa-1}{C_{p}} \frac{\Delta H^{2}}{R T^{2}} \exp (\Delta S / R) \exp (-\Delta H / R T) .
$$

The temperature dependence of $\mu_{\max }$ is mainly determined by the exponential term $\exp (-\Delta H / R T)$. In addition it is permissible to neglect the relative variation of $(\kappa-1) / C_{p}$ with temperature. Hence $\Delta H$ can be calculated from the slope of the plot of $\log \left(T^{2} \mu_{\max }\right)$ versus $1 / T$. In practice, it often happens that values of $C_{p}$ and $\kappa$ are not available over the range of temperature for which ultrasonic absorption results are available. In Fig. 3 there is presented the relaxation processes caused by isomerism in 1,1,2-trichloroethane [15].

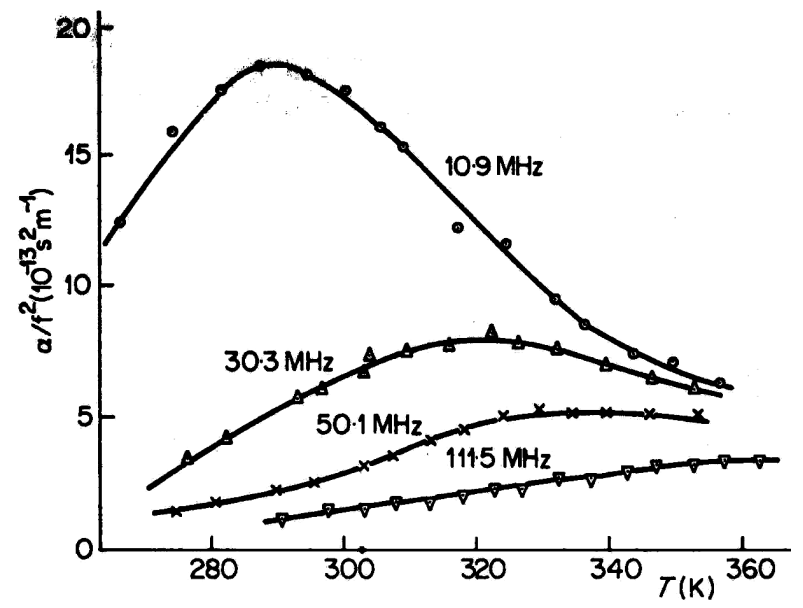

Fig. 3. Ultrasonic absorption versus temperature in 1,1,2-trichloroethane $-\Delta H_{b}=24 \mathrm{~kJ} / \mathrm{mol}, \Delta S_{b}=$ $11 \mathrm{~J} /(\mathrm{K} \mathrm{mol}), \Delta H=9 \mathrm{~kJ} / \mathrm{mol}[20]$.

\section{Measurement systems}

All the setups used for the acoustical spectroscopic measurements in chemical compounds will be described briefly, as they are a standard in acoustic spectroscopy.

\subsection{Pulse technique (10-3600 $\mathrm{MHz})$}

The amplitude absorption coefficient, $\alpha / f^{2}$, of an ultrasonic wave usually is measured with the pulse method [17-19] in two measuring setups covering frequency ranges from 10 to several hundreds $\mathrm{MHz}$. A modernized ultrasonic spectrometer designed by Lezhnev [6] enables to use a range up to several GHz. In this system the method of non-resonance excitation [21] of a lithium niobate monocrystal in a resonance chamber is used. The principle of operation of this system, presented in Fig. 4, is as follows: the two-channel pulse generator - 1 , switches on the oscilloscope time base 2 , the pulse generator -4 , and modulates generator signals - 3. A pulse with a determined carrier frequency leaves the high frequency generator and enters the coaxial quarter-wave resonator $\mathrm{R}_{1}$ through the coupling loop $P_{1}$. A quarter of the length of an electromagnetic wave is saved in the resonator cavity. The resonance frequency can be smoothly changed with the tuner in a range from 400 to $3600 \mathrm{MHz}$. A $5 \mathrm{~mm}$ in radius and $15 \mathrm{~mm}$ in length lithium niobate crystal (I) is inserted in the centre of the resonant cavity, so one end is within the range of the variable electric field. When the field in the resonator is excited by pulses, a pulse of mechanical vibrations is generated on the surface of the crystal, propagates along it and enters the investigated liquid [22]. (The $\mathrm{R}_{1}$ resonator together with the transducer are attached to the mobile part of the cathetometer, shifted with a $5 \times 10^{-7} \mathrm{~m}$ accuracy, in order to regulate the acoustic path in the liquid.) After passing through the liquid, the acoustic pulse enters transducer (II), where it is again changed into an electric pulse, which is fed into the input of the receiving amplifier 7 through resonator $R_{2}$ and coupling loop $P_{2}$ and then, sent directly to oscilloscope 2 .

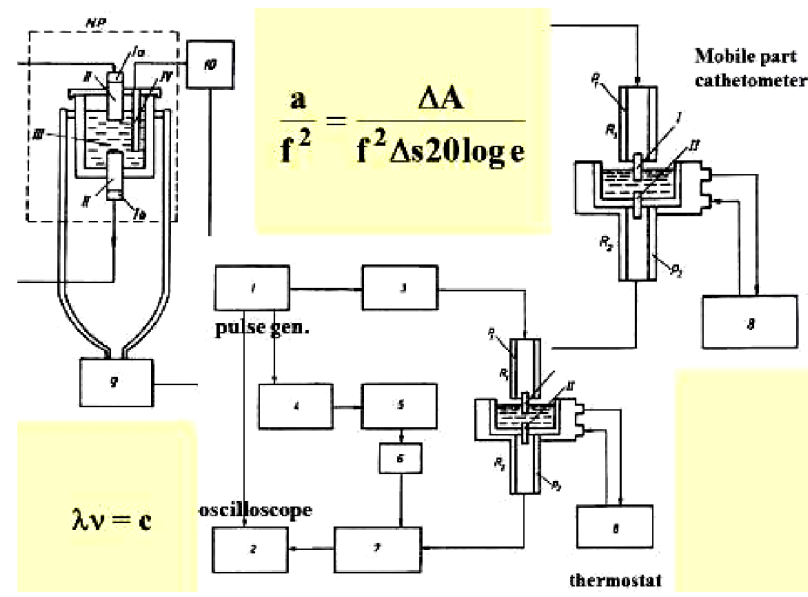

Fig. 4. Block diagram of a system for measuring absorption of ultrasonic waves in a frequency range 300 $3600 \mathrm{MHz}$

The mentioned above pulse generator 4 (acting as a regulated delay line and cutting out the unnecessary initial part of the pulse, enables a more legible oscilloscope visualization) switches on the calibrated pulse generator 5. Generator 5 sends a pulse through a damper (regulated smoothly from 0 to $30 \mathrm{~dB}$ with a $0.1 \mathrm{~dB}$ accu- 
racy) to oscilloscope 2 . This pulse is used in the absolute measurement of the amplitude absorption coefficient. Comparing amplitudes of two signals: ultrasonic, $A_{1}$, and calibrated, $A_{2}$, the value of the absorption coefficient, expressed in $\left[\mathrm{s}^{2} / \mathrm{m}\right]$, can be determined according to equation

$$
\frac{a}{f^{2}}=\frac{\Delta A}{f^{2} \Delta s 20 \log \mathrm{e}} .
$$

Estimated errors of determining the absorption coefficient are in a range from $3.5 \%$ for the frequency of $400 \mathrm{MHz}$ to $5 \%$ for $3600 \mathrm{MHz}$.

Measurements of the propagation velocity of ultrasound is performed in order to calculate classical absorption coefficients. For these measurements an ultrasonic pulse-phase interferometer is usually used [23, 24]. The values are calculated using the formula: $c=\lambda f$.

\subsection{Ultrasonic resonator (Eggers) method (200 $\mathrm{kHz}-5 \mathrm{MHz}$ )}

The measurements in low frequency range are provided by a resonance and methods. An ultrasonic resonance method has been introduced by Eggers [25-27]. It employs standing sound waves in a cylindrical cavity and allows the determination of velocity and relative attenuation in liquids as a function of frequency and bandwidth of characteristic resonance peaks. A brief outline of the resonator method will be given with the construction of the cell which is often used. Figure 5 shows a block diagram of the resonance method. The resonator consists of a liquid volume $V$ between two quartz plates used as transducers. A tuneable sine wave oscillator drives the input quartz transducer $\mathrm{Q}_{1}$ and produces a standing wave sound field in the liquid at characteristic frequencies $f_{n}$. At these frequencies the output quartz $\mathrm{Q}_{2}$ (the same type as $\mathrm{Q}_{1}$ ) delivers pronounced voltage peaks (Fig. 6), which after amplification by a tuneable receiver can be measured and displayed, in the sweep mode, on an oscilloscope screen (everything was achieved using the HewlettPackard 3585A Spectrum-Analyzer with the computer HP 9825).

The fundamental frequency of the liquid column $f_{L}$ is

$$
f_{L}=\frac{c_{L}}{2 x},
$$

where $c_{L}-$ ultrasound velocity in a liquid, $x-$ distance between the transducer.

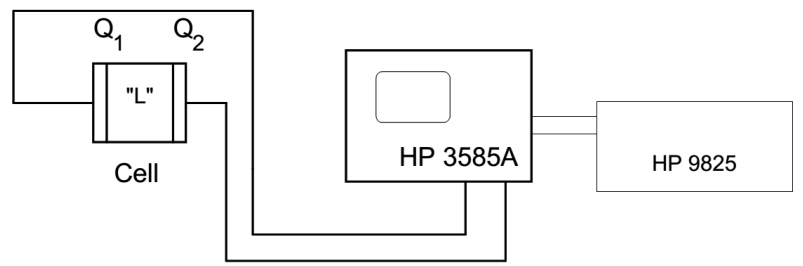

Fig. 5. Block diagram for the resonator method: $\mathrm{Q}_{1}$ - input, quartz transducer, $\mathrm{Q}_{2}$ - output, $\mathrm{L}$ - liquid in the cell.

For small attenuation values, $\alpha x \ll 1$. In this case it is possible to write the well known relation between half-
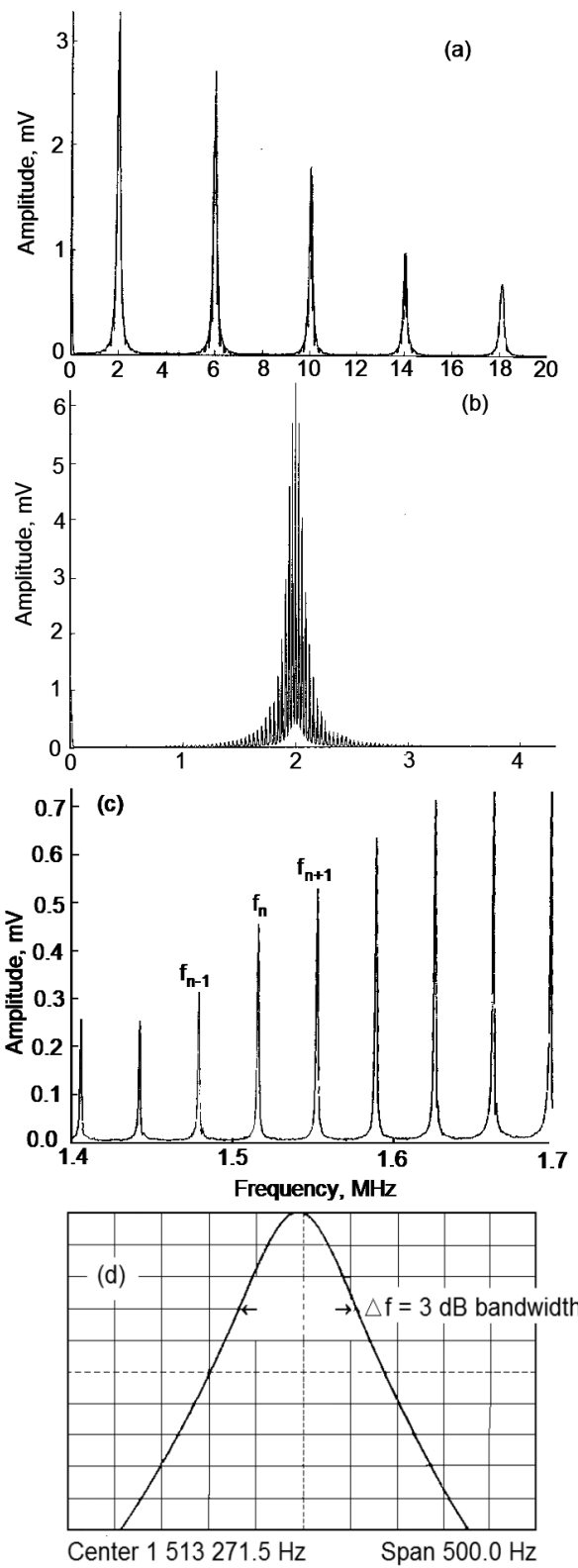

Fig. 6. Output voltage from the cell as a function of frequency: (a) the frequency span of $20 \mathrm{MHz}$, centre frequency $10 \mathrm{MHz}$. The maxima correspond to the fundamental frequency of the quartz transducer and its harmonics; (b) the frequency span of $4 \mathrm{MHz}$. The maximum corresponds to the fundamental frequency of the quartz $2 \mathrm{MHz}$, the others are the harmonics of the cell. The first on the left is fundamental frequency of the cell; (c) the frequency span of $0.5 \mathrm{MHz}$. The maxima correspond to the harmonic frequencies of the cell. $\Delta f=f_{n}-f_{n+1}$ corresponds to the fundamental frequency of the cell; (d) the frequency span of $500 \mathrm{~Hz}$.

power bandwidth (HPB) or "3 $\mathrm{dB}$ bandwidth" $\Delta f$ of a particular peak and the frequency $f_{n}$ of that peak,

$$
\frac{\alpha \lambda}{\pi}=\frac{\Delta f}{f_{n}}=\frac{1}{Q} .
$$

Equation (18) yields the quality factor $Q$ of an ideal res- 
onator with liquid attenuation only. The quality factor of the real resonator system $Q^{\text {real }}$ is inversely proportional to the total energy loss in the resonator system, which contains contribution from all kinds of energy loss in the cell such as liquid attenuation and additional losses from beam divergence, scattering, friction effects, imperfect reflection at the quartz surface, transducer mounting and coupling losses. If we assume all these energy loses are additive, then the overall quality factor $Q^{\text {real }}$ is given by

$$
\frac{1}{Q^{\text {real }}}=\frac{1}{Q^{\text {liquid }}}+\frac{1}{Q^{\text {extra }}},
$$

where $Q^{\text {liquid }}$ - quality factor due to sound absorption in the liquid, $Q^{\text {extra }}$ summarizes all additional energy losses in the cell mentioned before.

The excess absorption in a liquid caused by the relaxation of a chemical system is obtained by means of a reference measurement in the same call at the same frequencies with a suitable reference liquid (which eliminates other attenuation effects). This reference liquid should have - especially for the lower frequency range - equal or very similar sound velocity to insure an identical sound field pattern in the cell for both measurements. The excess absorption per wavelength $(\alpha \lambda)^{\text {excess }}$ in the liquid is obtained from Eqs. (20) and (21):

$$
\begin{aligned}
& (\alpha \lambda)^{\text {excess }}=\alpha \lambda-(\alpha \lambda)^{\mathrm{ref}}=\pi\left[\frac{\Delta f_{s}-\Delta f^{\mathrm{ref}}}{f_{n}}\right] \\
& \alpha \lambda \approx(\alpha \lambda)^{\mathrm{ref}}+\pi\left[\left(\frac{\Delta f_{n}}{f_{n}}\right)-\left(\frac{\Delta f_{n}}{f_{n}}\right)^{\mathrm{ref}}\right],
\end{aligned}
$$

where $\Delta f_{s}$ and $\Delta f^{\text {ref }}$ - corresponding HPB values of the $n$-th resonance peak in the sample and reference liquid, respectively. This simple relation holds only if subsequent resonance peaks are very well separated, i.e., if $\Delta f \ll \delta f=f_{n}-f_{n+1}$.

The fundamental frequency $f_{L}$ of the liquid column can be determined with the rough approximation by the formula (22):

$$
f_{L} \approx f_{n}-f_{n+1} \text {. }
$$

Close to $\lambda / 4$ frequencies of the transducer, namely $f_{Q} / 2$, $3 f_{Q} / 2,5 f_{Q} / 2$, etc. where the air backing of quartz is transformed to a very high impedance at the quartzliquid interface we can write the relation

$$
\frac{c}{c^{\mathrm{ref}}}=\frac{\delta f}{\delta f^{\mathrm{ref}}}\left[1+2 \frac{\delta f Z-\delta f^{\mathrm{ref}} Z^{\mathrm{ref}}}{f_{Q} Z_{Q}}\right],
$$

where $c^{\text {ref }}$ and $\delta f-$ measured values for reference liquid.

\section{Resume}

It is necessary to take into account that for very precise ultrasonic investigations of fluids and liquid crystals it is indispensable to possess several spectrometers in very wide frequency range. For supplementation of these molecular investigations it is very useful to include the photoacoustic research [28-30], as well as pressure dependence of relaxation $[12,13]$.

\section{References}

[1] G. Stokes, Camb. Trans. Philos. Soc. 8, 287 (1845).

[2] G. Kirchhoff, Ann. Phys. 134, 177 (1868).
[3] B.A. Prokofiev, Izv. Acad. Sci. Sov. Union 7, 94 (1957) (in Russian).

[4] I.G. Mikhaylov, W.A. Soloviev, Yu.P. Syrnikov, Fundamentals of molecular acoustics, Nauka, 1964 (in Russian).

[5] W.P. Mason, Physical Acoustics, Vol. II, Part A, Properties of Gases, Liquids, and Solutions, Academic Press, New York 1965.

[6] B.B.J. Linde, Acoustical Spectroscopy of Cyclic and Heterocyclic Compounds, Ketones and Polluted Water Surface, Wydawnictwo Uniwersytetu Gdańskiego, Gdańsk 1997.

[7] B.B.J. Linde, N.B. Lezhnev, J. Mol. Struct. 754 , 111 (2005).

[8] B.B.J. Linde, E. B. Skrodzka, N.B. Lezhnev, Int. J. Thermophys. 33, 664 (2012).

[9] B.B.J. Linde, N. B. Lezhnev, Ultrasonics 44, 1467 (2006).

[10] B.B.J. Linde, N.B. Lezhnev, Ultrasonics 36, 958 (1998).

[11] B.B.J. Linde, N.B. Lezhnev, Ultrasonics 38, 945 (2000).

[12] B.B.J. Linde, U.M. Esanov, J. Phys. IV 129, 55 (2005).

[13] B.B.J. Linde, U.M. Esanov, Instrument. Sci. Technol. 27, 447 (1999).

[14] S. Glasstone, K.J. Laidler, H. Eyring, Theory of Rate Processes, McGraw-Hill, New York 1941.

[15] M.S. De Groot, Ultrasonic Relaxation due to Rotational Isomerism, N.V. Drukkerij V/H.J.J. Groen \& Zn, Leiden 1958.

[16] A. J. Matheson, Molecular Acoustics, WileyInterscience, London 1971.

[17] J. Pellam, J.K. Galt, J. Chem. Phys. 14, 608 (1946).

[18] H.J. Mc Skimin, J. Acoust. Soc. Am. 29, 1185 (1957.

[19] J.A. Andreae, R. Bass, E.L. Heasell, J. Lamb, Acus tica 8, 131 (1958).

[20] J. Lamb, Z. Elektrochem. 64, 135 (1960).

[21] K. Baranskij, Dokl. Russ. Acad. Sci. 114, 517 (1957) (in Russian).

[22] R. Płowiec, Arch. Acoust. 4, 411 (1970).

[23] J. Wehr, Measurements of Absorption and Velocity of Ultrasound Waves, PWN, Warszawa 1972 (in Polish).

[24] V. Ilgunas, V. Yaronis, V. Sukackas, Ultrasonic Interferometers, Mokslas, Vilnius 1983 (in Russian).

[25] F. Eggers, Th. Funck, Rev. Sci. Instrum. 44, 969 (1973).

[26] F. Eggers, Th. Funck, K.H. Richmann, Acustica 40, 273 (1978).

[27] F. Eggers, Acustica 80, 397 (1994).

[28] A. Sikorska, D. Dadarlat, B.B.J. Linde, M. Streza, C. Neamtu, A. Sliwiński, J. Phys. IV (France) V 137, 341 (2006).

[29] A. Sikorska, B.B.J. Linde, Chem. Phys. 354, 148 (2008).

[30] A. Sikorska, B.J. Linde, J.I. Kukielski, J. Opt. A Pure Appl. Opt. 3, 71 (2001). 